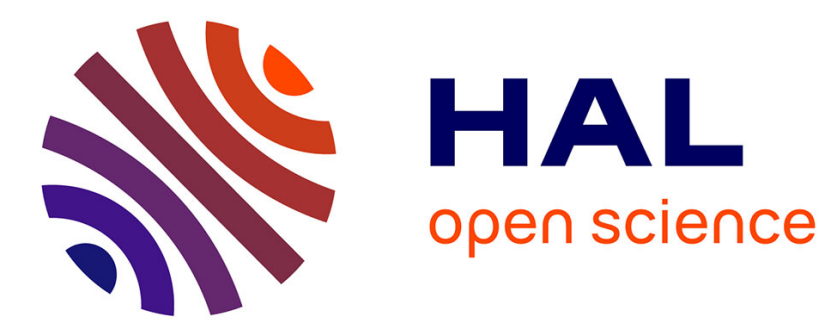

\title{
Separable L-embedded Banach spaces are unique preduals
}

Hermann Pfitzner

\section{To cite this version:}

Hermann Pfitzner. Separable L-embedded Banach spaces are unique preduals. Bull. Lond. Math. Soc., 2007, 39, pp.1039-1044. 10.1112/blms/bdm077 . hal-00007545

\section{HAL Id: hal-00007545 \\ https://hal.science/hal-00007545}

Submitted on 17 Jul 2005

HAL is a multi-disciplinary open access archive for the deposit and dissemination of scientific research documents, whether they are published or not. The documents may come from teaching and research institutions in France or abroad, or from public or private research centers.
L'archive ouverte pluridisciplinaire HAL, est destinée au dépôt et à la diffusion de documents scientifiques de niveau recherche, publiés ou non, émanant des établissements d'enseignement et de recherche français ou étrangers, des laboratoires publics ou privés. 


\title{
A separable L-embedded Banach space has property $(\mathrm{X})$ and is therefore the unique predual of its dual. \\ H. Pfitzner
}

\begin{abstract}
In this note the following is proved. Separable L-embedded spaces - that is separable Banach spaces which are complemented in their biduals such that the norm between the two complementary subspaces is additive - have property $(\mathrm{X})$ which, by a result of Godefroy and Talagrand, entails uniqueness of the space as a predual.
\end{abstract}

We say that a Banach space $X$ is the unique predual of its dual (more precisely the unique isometric predual of its dual) in case it is isometric to any Banach space whose dual is isometric to the dual of $X$. (We say that two Banach spaces $Y$ and $Z$ are isomorphic if there is a bounded linear bijective operator $T: Y \rightarrow Z$ with bounded inverse $T^{-1}$; if moreover $\|T(y)\|=\|y\|$ for all $y \in Y$ we say that $Y$ and $Z$ are isometric.) In general a Banach space need not be the unique predual of its dual, for example $c$ and $c_{0}$ are not isometric Banach spaces although their duals are.

As shown by Grothendieck [10, Rem. 4] in 1955, $L^{1}$-spaces are unique preduals of their duals. Using essentially a result of Dixmier [5] from 1953, Sakai [19, Cor. 1.13.3] observed that more generally preduals of von Neumann algebras are unique, and Barton and Timoney [2] and Horn [13] generalized this to preduals of $J B W^{*}$ triples. Ando [1] stated the uniqueness as a predual for the quotient $L^{1} / H_{0}^{1}$.

As Banach spaces these examples have in common to be L-summands in their biduals or, for short, to be L-embedded. By definition a Banach space $X$ is Lembedded if there is a projection $P$ on its bidual $X^{* *}$ with range $X$ such that $\left\|P x^{* *}\right\|+\left\|x^{* *}-P x^{* *}\right\|=\left\|x^{* *}\right\|$ for all $x^{* *} \in X^{* *}$.

The standard reference for L-embedded spaces is [11], for a survey on unique preduals we refer to [8], for general Banach space theory to [14], [15], or [4].

If not stated otherwise a sequence $\left(z_{j}\right)$ (and similarly a series $\sum z_{j}$ ) is indexed by $\mathbb{N}=\{1,2, \ldots\}$; we write $\mathbb{N}_{0}=\{0,1,2, \ldots\}$. Recall that a series $\sum z_{j}$ in a Banach space $Z$ is called weakly unconditionally Cauchy (wuC for short) if $\sum\left|z^{*}\left(z_{j}\right)\right|$ converges for each $z^{*} \in Z^{*}$ or, equivalently, if there is a number $M$ such that $\left\|\sum_{j=1}^{n} \alpha_{j} z_{j}\right\| \leq M \max _{1 \leq j \leq n}\left|\alpha_{j}\right|$ for all $n \in \mathbb{N}$ and all scalars $\alpha_{j}$. It is well known by a result of Bessaga and Pełczyński that a Banach space contains a subspace isomorphic to $c_{0}$ if and only if it contains a wuC-series $\sum z_{j}$ such that inf $\left\|z_{j}\right\|>0$. In case $\sum x_{j}^{*}$ is a wuC-series in a dual Banach space $X^{*}$ we denote the $w^{*}$-limit (that is the limit in the $\sigma\left(X^{*}, X\right)$-topology) of the sequence $\left(\sum_{j=1}^{n} x_{j}^{*}\right)$ by $\sum^{*} x_{j}^{*}$.

In their study of unique preduals Godefroy and Talagrand [9] defined

Definition 1 (Property (X)) A Banach space $X$ has property $(X)$ if for each $x^{* *} \in X^{* *} \backslash X$ there exists a wuC-series $\sum x_{j}^{*}$ in $X^{*}$ such that

$$
\sum x^{* *}\left(x_{j}^{*}\right) \neq x^{* *}\left(\sum^{*} x_{j}^{*}\right) .
$$


They proved

Theorem 2 (Godefroy, Talagrand) A Banach space $X$ with property $(X)$ is the unique predual of its dual.

Moreover, every $x^{* *} \in X^{* *}$ which is strongly Baire mesurable on $\left(X^{*}, w^{*}\right)$ (for the definition see [8, Th. V.3]) - in particular, every $x^{* *} \in X^{* *}$ which is Borel on $\left(X^{*}, w^{*}\right)$ - belongs to $X$.

Up to now it has been known that a class of L-embedded spaces, namely the duals of M-embedded spaces (see [11] for the definiton), have property (X) [11, p. 148]. Furthermore it has been known ([17], [11, Th. VI.2.7]) that L-embedded spaces have Pełczyński's property $\left(\mathrm{V}^{*}\right)$; the latter one is similar to and implied by property (X) but is, by an example of Talagrand [20], strictly weaker than (X).

In view of all this it was natural to ask whether L-embedded spaces are unique preduals and have property $(\mathrm{X})$ (see [11, Problem page 123]). At least in the separable case the answer is yes.

Theorem 3 Separable L-embedded Banach spaces have property (X).

Proof: Let $X$ be L-embedded and $P$ be the corresponding projection on $X^{* *}$ with range $X$; we put $Q=\operatorname{id}_{X^{* *}}-P$. Denoting the range of $Q$ by $X_{\mathrm{s}}$ we have the decomposition $X^{* *}=X \oplus_{1} X_{\mathrm{s}}$. Let the sequence $\left(x_{n}\right)$ be dense in $X$. Let $x^{* *} \in$ $X^{* *} \backslash X$. Let $\eta=\left\|x_{\mathrm{s}}\right\|$ where $x^{* *}=x+x_{\mathrm{s}}, x \in X, x_{\mathrm{s}} \in X_{\mathrm{s}}$. We have that $\eta>0$ because $x^{* *} \notin X$.

Let $1>\varepsilon>0$. By the Bishop-Phelps theorem [3], [12] there are $x^{*} \in X^{*}$ and $\tilde{x}^{* *} \in X^{* *}$ such that $\left\|x^{*}\right\|=1$ and $\left\|\tilde{x}^{* *}-x^{* *}\right\|<\varepsilon \eta / 3$ and such that $\tilde{x}^{* *}$ attains its norm on $x^{*}$ that is $\tilde{x}^{* *}\left(x^{*}\right)=\left\|\tilde{x}^{* *}\right\|$. For the decomposition $\tilde{x}^{* *}=\tilde{x}+\tilde{x}_{\mathrm{s}}$ we have

$$
\tilde{x}_{\mathrm{s}}\left(x^{*}\right)=\left\|\tilde{x}_{\mathrm{s}}\right\| \quad \text { and } \quad\left\|\tilde{x}_{\mathrm{s}}-x_{\mathrm{s}}\right\|<\varepsilon \eta / 3
$$

because $\left\|\tilde{x}_{\mathrm{s}}\right\| \geq\left|\tilde{x}_{\mathrm{s}}\left(x^{*}\right)\right|=\left|\tilde{x}^{* *}\left(x^{*}\right)-x^{*}(\tilde{x})\right| \geq\left\|\tilde{x}^{* *}\right\|-\|\tilde{x}\|=\left\|\tilde{x}_{\mathrm{s}}\right\|$ and $\varepsilon \eta / 3>$ $\left\|\tilde{x}^{* *}-x^{* *}\right\|=\left\|\tilde{x}-x+\tilde{x}_{\mathrm{s}}-x_{\mathrm{s}}\right\|=\|\tilde{x}-x\|+\left\|\tilde{x}_{\mathrm{s}}-x_{\mathrm{s}}\right\|$.

Choose a sequence $\left(\varepsilon_{j}\right)$ of strictly positive numbers such that $\prod_{j=1}^{\infty}\left(1+\varepsilon_{j}\right)<1+\varepsilon$ and $\prod_{j=1}^{\infty}\left(1-\varepsilon_{j}\right)>1-\varepsilon$.

By induction over $\mathbb{N}_{0}$ we construct two sequences $\left(x_{n}^{*}\right)_{n \in \mathbb{N}_{0}}$ and $\left(y_{n}^{*}\right)_{n \in \mathbb{N}_{0}}$ in $X^{*}$ (of which the first members $x_{0}^{*}$ and $y_{0}^{*}$ are auxiliary values used only for the induction) such that, for all (real or complex) scalars $\alpha_{j}$, the following holds:

$$
\begin{aligned}
x_{0}^{*}=0, & \left\|y_{0}^{*}\right\|=1, \\
y_{n}^{*} & =x^{*}-\sum_{j=0}^{n} x_{j}^{*} \\
\left(\prod_{j=1}^{n}\left(1-\varepsilon_{j}\right)\right) \max _{0 \leq j \leq n}\left|\alpha_{j}\right| \leq\left\|\alpha_{0} y_{n}^{*}+\sum_{j=1}^{n} \alpha_{j} x_{j}^{*}\right\| & \leq\left(\prod_{j=1}^{n}\left(1+\varepsilon_{j}\right)\right) \max _{0 \leq j \leq n}\left|\alpha_{j}\right|, \quad \text { if } n \geq 1,
\end{aligned}
$$




$$
\begin{array}{ll}
\tilde{x}_{\mathrm{s}}\left(x_{j}^{*}\right)=0 & \text { if } 0 \leq j \leq n, \\
x_{\mathrm{s}}\left(x_{j}^{*}\right)=0 & \text { if } 0 \leq j \leq n, \\
y_{n}^{*}\left(x_{k}\right)=0 & \text { if } 1 \leq k \leq n .
\end{array}
$$

For $n=0$ we set $x_{0}^{*}=0$ and $y_{0}^{*}=x^{*}$.

We notice that the restriction of $P^{*}$ to $X^{*}$ is an isometric isomorphism from $X^{*}$ onto $X_{\mathrm{s}}^{\perp}$, that $Q$ is a contractive projection and that $X^{* * *}=X_{\mathrm{s}}^{\perp} \oplus_{\infty} X^{\perp}$.

For the induction step $n \mapsto n+1$ suppose now that $x_{0}^{*}, \ldots, x_{n}^{*}$ and $y_{0}^{*}, \ldots, y_{n}^{*}$ have been constructed. Put

$$
\begin{aligned}
& E=\operatorname{lin}\left(\left\{x^{*}, x_{0}^{*}, \ldots, x_{n}^{*}, y_{n}^{*}, P^{*} x_{0}^{*}, \ldots, P^{*} x_{n}^{*}, P^{*} y_{n}^{*}\right\}\right) \subset X^{* * *} \\
& F=\operatorname{lin}\left(\left\{x_{1}, \ldots, x_{n+1}, x_{\mathrm{s}}, \tilde{x}_{\mathrm{s}}\right\}\right) \subset X^{* *}
\end{aligned}
$$

Note that $Q^{*} x_{j}^{*}, Q^{*} y_{n}^{*} \in E$ for $0 \leq j \leq n$. By the principle of local reflexivity there is an operator $R: E \rightarrow X^{*}$ such that

$$
\begin{aligned}
\left(1-\varepsilon_{n+1}\right)\left\|e^{* * *}\right\| & \leq\left\|R e^{* * *}\right\| \leq\left(1+\varepsilon_{n+1}\right)\left\|e^{* * *}\right\|, \\
f^{* *}\left(R e^{* * *}\right) & =e^{* * *}\left(f^{* *}\right), \\
R_{\mid E \cap X^{*}} & =\operatorname{id}_{E \cap X^{*}}
\end{aligned}
$$

for all $e^{* * *} \in E$ and $f^{* *} \in F$.

We define

$$
x_{n+1}^{*}=R P^{*} y_{n}^{*} \quad \text { and } \quad y_{n+1}^{*}=R Q^{*} y_{n}^{*} .
$$

First we notice that $(3, n+1)$ holds because

$$
x^{*}-\sum_{j=0}^{n+1} x_{j}^{*} \stackrel{(3)}{=} y_{n}^{*}-x_{n+1}^{*}=R\left(y_{n}^{*}-P^{*} y_{n}^{*}\right)=R Q^{*} y_{n}^{*}=y_{n+1}^{*} .
$$

In the following we use the convention $\sum_{j=1}^{0}(\cdots)=0$. Then we have that

$$
\alpha_{0} y_{n+1}^{*}+\sum_{j=1}^{n+1} \alpha_{j} x_{j}^{*}=R\left(Q^{*}\left(\alpha_{0} y_{n}^{*}+\sum_{j=1}^{n} \alpha_{j} x_{j}^{*}\right)+P^{*}\left(\alpha_{n+1} y_{n}^{*}+\sum_{j=1}^{n} \alpha_{j} x_{j}^{*}\right)\right) .
$$

The second inequality of $(4, n+1)$ can be seen as follows:

$$
\begin{aligned}
\left\|\alpha_{0} y_{n+1}^{*}+\sum_{j=1}^{n+1} \alpha_{j} x_{j}^{*}\right\| & \stackrel{(8)}{\leq}\left(1+\varepsilon_{n+1}\right)\left\|Q^{*}\left(\alpha_{0} y_{n}^{*}+\sum_{j=1}^{n} \alpha_{j} x_{j}^{*}\right)+P^{*}\left(\alpha_{n+1} y_{n}^{*}+\sum_{j=1}^{n} \alpha_{j} x_{j}^{*}\right)\right\| \\
& =\left(1+\varepsilon_{n+1}\right) \max \left\{\left\|Q^{*}\left(\alpha_{0} y_{n}^{*}+\sum_{j=1}^{n} \alpha_{j} x_{j}^{*}\right)\right\|,\left\|P^{*}\left(\alpha_{n+1} y_{n}^{*}+\sum_{j=1}^{n} \alpha_{j} x_{j}^{*}\right)\right\|\right\} \\
& \leq\left(1+\varepsilon_{n+1}\right) \max \left\{\left\|\alpha_{0} y_{n}^{*}+\sum_{j=1}^{n} \alpha_{j} x_{j}^{*}\right\|,\left\|\alpha_{n+1} y_{n}^{*}+\sum_{j=1}^{n} \alpha_{j} x_{j}^{*}\right\|\right\} \\
& \leq\left(\prod_{j=1}^{n+1}\left(1+\varepsilon_{j}\right)\right) \max \left\{\max _{0 \leq j \leq n}\left|\alpha_{j}\right|, \max _{1 \leq j \leq n+1}\left|\alpha_{j}\right|\right\} \\
& =\left(\prod_{j=1}^{n+1}\left(1+\varepsilon_{j}\right) \max _{0 \leq j \leq n+1}\left|\alpha_{j}\right|\right.
\end{aligned}
$$


where the last inequality comes from (2) if $n=0$ and from (4) if $n \geq 1$.

For the first inequality of $(4, n+1)$ we estimate

$$
\begin{aligned}
\left\|\alpha_{0} y_{n+1}^{*}+\sum_{j=1}^{n+1} \alpha_{j} x_{j}^{*}\right\| & \stackrel{(8)}{\geq}\left(1-\varepsilon_{n+1}\right)\left\|Q^{*}\left(\alpha_{0} y_{n}^{*}+\sum_{j=1}^{n} \alpha_{j} x_{j}^{*}\right)+P^{*}\left(\alpha_{n+1} y_{n}^{*}+\sum_{j=1}^{n} \alpha_{j} x_{j}^{*}\right)\right\| \\
& =\left(1-\varepsilon_{n+1}\right) \max \left\{\left\|Q^{*}\left(\alpha_{0} y_{n}^{*}+\sum_{j=1}^{n} \alpha_{j} x_{j}^{*}\right)\right\|,\left\|\alpha_{n+1} y_{n}^{*}+\sum_{j=1}^{n} \alpha_{j} x_{j}^{*}\right\|\right\}
\end{aligned}
$$

in case $\left|\alpha_{0}\right|=\max _{0 \leq j \leq n+1}\left|\alpha_{j}\right|$ we observe that $Q \tilde{x}_{\mathrm{s}}=\tilde{x}_{\mathrm{s}}$, that $\tilde{x}_{\mathrm{s}}\left(y_{n}^{*}\right)=\tilde{x}_{\mathrm{s}}\left(x^{*}\right)-$ $\sum_{j=1}^{n} \tilde{x}_{\mathrm{s}}\left(x_{j}^{*}\right)=\tilde{x}_{\mathrm{s}}\left(x^{*}\right)$ by $(5)$, and we continue the estimate by

$$
\begin{aligned}
\cdots & \geq\left(1-\varepsilon_{n+1}\right)\left|\left(Q^{*}\left(\alpha_{0} y_{n}^{*}+\sum_{j=1}^{n} \alpha_{j} x_{j}^{*}\right)\right)\left(\frac{\tilde{x}_{\mathrm{s}}}{\left\|\tilde{x}_{\mathrm{s}}\right\|}\right)\right| \\
& =\frac{\left(1-\varepsilon_{n+1}\right)}{\left\|\tilde{x}_{\mathrm{s}}\right\|}\left|\tilde{x}_{\mathrm{s}}\left(\alpha_{0} y_{n}^{*}+\sum_{j=1}^{n} \alpha_{j} x_{j}^{*}\right)\right| \\
& \stackrel{(5)}{=} \frac{\left(1-\varepsilon_{n+1}\right)}{\left\|\tilde{x}_{\mathrm{s}}\right\|}\left|\alpha_{0}\right|\left|\tilde{x}_{\mathrm{s}}\left(y_{n}^{*}\right)\right|=\frac{\left(1-\varepsilon_{n+1}\right)}{\left\|\tilde{x}_{\mathrm{s}}\right\|}\left|\alpha_{0}\right|\left|\tilde{x}_{\mathrm{s}}\left(x^{*}\right)\right| \\
& =\left(1-\varepsilon_{n+1}\right)\left|\alpha_{0}\right|
\end{aligned}
$$

whereas in case $\left|\alpha_{0}\right| \neq \max _{0 \leq j \leq n+1}\left|\alpha_{j}\right|$ we get

$$
\begin{aligned}
\cdots & \geq\left(1-\varepsilon_{n+1}\right)\left\|\alpha_{n+1} y_{n}^{*}+\sum_{j=1}^{n} \alpha_{j} x_{j}^{*}\right\| \\
& \geq\left(\prod_{j=1}^{n+1}\left(1-\varepsilon_{j}\right)\right) \max _{1 \leq j \leq n+1}\left|\alpha_{j}\right|
\end{aligned}
$$

where the last inequality comes from (2) if $n=0$ and from (4) if $n \geq 1$. Thus we obtain the first inequality of $(4, n+1)$.

The conditions $(5, n+1),(6, n+1)$ and $(7, n+1)$ are easy to verify because $P x_{\mathrm{s}}=P \tilde{x}_{\mathrm{s}}=Q x_{k}=0$ thus

$$
\begin{aligned}
& x_{\mathrm{s}}\left(x_{n+1}^{*}\right)=x_{\mathrm{s}}\left(R P^{*} y_{n}^{*}\right)=\left(P^{*} y_{n}^{*}\right)\left(x_{\mathrm{s}}\right)=P x_{\mathrm{s}}\left(y_{n}^{*}\right)=0, \\
& \tilde{x}_{\mathrm{s}}\left(x_{n+1}^{*}\right)=\tilde{x}_{\mathrm{s}}\left(R P^{*} y_{n}^{*}\right)=\left(P^{*} y_{n}^{*}\right)\left(\tilde{x}_{\mathrm{s}}\right)=P \tilde{x}_{\mathrm{s}}\left(y_{n}^{*}\right)=0, \\
& y_{n+1}^{*}\left(x_{k}\right)=\left(R Q^{*} y_{n}^{*}\right)\left(x_{k}\right)=y_{n}^{*}\left(Q x_{k}\right)=0 .
\end{aligned}
$$

This ends the induction.

By (4), $\sum x_{j}^{*}$ is wuC (where, as indicated above in the introduction, $j$ runs through $\mathbb{N}$ ). We have that $\sum^{*} x_{j}^{*}=x^{*}$ by (3) (and (2)) because by (7) and the density of the $x_{k}$ the $w^{*}$-limit of $\left(y_{n}^{*}\right)$ is 0 . This easily entails (1) because we have $\sum x_{\mathrm{s}}\left(x_{j}^{*}\right)=0$ by $(6)$, we have $\left\|\tilde{x}_{\mathrm{s}}\right\| \geq\left\|x_{\mathrm{s}}\right\|-\left\|x_{\mathrm{s}}-\tilde{x}_{\mathrm{s}}\right\|>(1-\varepsilon / 3) \eta$ and trivially $\left(\sum^{*} x_{j}^{*}\right)(x)=\sum x_{j}^{*}(x)$ thus

$$
x^{* *}\left(\sum^{*} x_{j}^{*}\right)-\sum x^{* *}\left(x_{j}^{*}\right)=x_{\mathrm{s}}\left(\sum^{*} x_{j}^{*}\right)-\sum x_{\mathrm{s}}\left(x_{j}^{*}\right)
$$




$$
\begin{aligned}
& =x_{\mathrm{s}}\left(x^{*}\right)=\left\|\tilde{x}_{\mathrm{s}}\right\|-\left(\tilde{x}_{\mathrm{s}}-x_{\mathrm{s}}\right)\left(x^{*}\right) \\
& >\left(1-\frac{\varepsilon}{3}\right) \eta-\frac{\varepsilon \eta}{3} \\
& >(1-\varepsilon)\left\|Q x^{* *}\right\|>0 .
\end{aligned}
$$

This ends the proof.

We have already mentioned Godefroy's and Talagrand's result that property (X) implies the uniqueness of a Banach space as a predual; moreover, since (X) is hereditary and stable by equivalent norms we obtain

Corollary 4 A Banach space that is isomorphic to a subspace of a separable $L$ embedded space is the unique predual of its dual.

Remarks:

1. It follows immediately from the first variant of the proof of theorem 3 that if $\tilde{x}_{\mathrm{s}}$ is a non zero norm attaining element of $X_{\mathrm{s}}$ then the two expressions in (1) differ by the greatest possible value, more precisely

$$
\tilde{x}_{\mathrm{s}}\left(\sum^{*} x_{j}^{*}\right)=\left\|\tilde{x}_{\mathrm{s}}\right\| \neq 0=\sum \tilde{x}_{\mathrm{s}}\left(x_{j}^{*}\right)
$$

with $\left\|\sum^{*} x_{j}^{*}\right\|=1$.

2. Is it possible to refine the proof of theorem 3 so to produce a sequence spanning $c_{0}$ almost or asymptotically isometrically? We say that a sequence $\left(z_{j}\right)$ in a Banach space $Z$ spans $c_{0}$ almost isometrically if there exists a sequence $\left(\delta_{m}\right)$ satisfying $[0,1[\ni$ $\delta_{m} \rightarrow 0$ such that $\left(1-\delta_{m}\right) \sup _{m \leq j \leq n}\left|\alpha_{j}\right| \leq\left\|\sum_{j=m}^{n} \alpha_{j} z_{j}\right\| \leq\left(1+\delta_{m}\right) \sup _{m \leq j \leq n}\left|\alpha_{j}\right|$ for all $m \leq n$. If we have even $\sup _{j \leq n}\left(1-\delta_{j}\right)\left|\alpha_{j}\right| \leq\left\|\sum_{j=1}^{n} \alpha_{j} z_{j}\right\| \leq \sup _{j \leq n}(1+$ $\left.\delta_{j}\right)\left|\alpha_{j}\right|$ for all $n \in \mathbb{N}$ then $\left(z_{j}\right)$ is said to span $c_{0}$ asymptotically (or asymptotically isometrically). While, by James' distortion theorem, a Banach space isomorphic to $c_{0}$ always contains an almost isomorphic copy of $c_{0}$, Dowling, Johnson, Lennard and Turett [6] proved the existence of a $c_{0}$-copy which does not contain asymptotic copies of $c_{0}$. Note that the L-structure of an L-embedded Banach space and, respectively, the M-structure of its dual have an influence on the existance of asymptotic copies of $\ell^{1}$ and, respectively, $c_{0}$. For example, it has been proved in [18] that each almost isometric copy of $\ell^{1}$ inside an L-embedded space contains an asymtotic copy of $\ell^{1}$ (see [18] also for the definitions) and it has been proved there that if an L-embedded space is the dual of an M-embedded space then its dual contains asymptotic copies of $c_{0}$.

3. There is an interesting difference of the construction of a $c_{0}$-copy in the present proof and in the proof of property $\left(\mathrm{V}^{*}\right)$. The latter one works for both separable and non-separable L-embedded spaces whereas the present proof of property (X) runs into an obstacle in the non-separable case: Edgar [7, Prop. 12] showed that $\ell^{1}(\Gamma)$ has property $(\mathrm{X})$ if and only if $\operatorname{card}(\Gamma)$ is not a real measurable cardinality (that is if and only if there is no non-zero measure on $\Gamma$ vanishing on singletons). For a discussion of $(\mathrm{X})$ and measurable cardinals we refer to [16]. It seems reasonable to conjecture that an L-embedded Banach space may have property $(\mathrm{X})$ if it does not 
contain a subspace isomorphic to $\ell^{1}(\Gamma)$ with $\operatorname{card}(\Gamma)$ measurable, or, perhaps, if it has a dense subset of non-measurable cardinality.

4. Given a Banach space $Z$ it might occur that its bidual contains an element $z_{0}^{* *}$ which is L-direct to $Z$ that is

$$
\left\|z+z_{0}^{* *}\right\|=\|z\|+\left\|z_{0}^{* *}\right\| \text { for all } z \in Z .
$$

Godefroy has shown ([11, IV.2] or [14, I.18.5.6]) that viewed as a function on the unit ball of the dual $\left(B_{Z^{*}}, w^{*}\right)$ such an element $z_{0}^{* *}$ is "very" discontinuous, for example it is nowhere continuous on $\left(B_{Z^{*}}, w^{*}\right)$. The space $Z=\mathrm{C}([0,1])$ and the function $z_{0}^{* *}=1_{\mathrm{IQ} \cap[0,1]}-1_{(\mathbb{R} \backslash \mathrm{IQ}) \cap[0,1]}$ serve as an example. This function $z_{0}^{* *}$ is of second Baire class but does not belong to $Z$. In other words, the "local" property (14) is definitely weaker than the "global" one of being L-embedded because if $Z$ were l-embedded then the second Baire class function $z_{0}^{* *}$ would belong to $Z$ (cf. the second part of theorem 2).

Acknowledgement I thank G. Godefroy and M. Neufang for interesting discussions, and Dirk Werner for not only spotting but also correcting a mistake in a previous version of the proof.

\section{References}

[1] T. Ando. On the predual of $H^{\infty}$. Commentationes Mathematicae. Special volume in honour of W. Orlicz, (1978) 33-40.

[2] T. Barton and R. M. Timoney. Weak*-continuity of Jordan triple products and its applications. Math. Scand., 59:177-191, 1986.

[3] E. Bishop and R. R. Phelps. A proof that every Banach space is subreflexive. Bull. Amer. Math. Soc., 67:97-98, 1961.

[4] J. Diestel. Sequences and Series in Banach Spaces. Springer, Berlin-HeidelbergNew York, 1984.

[5] J. Dixmier. Formes linéaires sur un anneau d'opérateurs. Bull. Soc. Math. France, 81:9-39, 1953.

[6] P. N. Dowling, W. B. Johnson, C. J. Lennard, and B. Turett. The optimality of James's distortion theorems. Proc. Amer. Math. Soc., 125:167-174, 1997.

[7] G. A. Edgar. An ordering for the Banach spaces. Pacific J. Math., 108:8398, 1983.

[8] G. Godefroy. Existence and uniqueness of isometric preduals: a survey. In BorLuh Lin, editor, Banach Space Theory. Proc. of the Iowa Workshop on Banach Space Theory 198\%, pages 131-193. Contemp. Math. 85, 1989. 
[9] G. Godefroy and M. Talagrand. Nouvelles classes d'espaces de Banach à predual unique. Séminaire d'Analyse Fonctionelle de l'Ecole Polytechnique, 1980-81.

[10] A. Grothendieck. Une caractérisation vectorielle-métrique des espaces $L^{1}$. Canadian J. Math., 7:552-561, 1955.

[11] P. Harmand, D. Werner, and W. Werner. M-ideals in Banach Spaces and Banach Algebras. Lecture Notes in Mathematics 1547. Springer, 1993.

[12] R. B. Holmes. Geometric Functional Analysis and its Applications. Springer, Berlin-Heidelberg-New York, 1975.

[13] G. Horn. Characterization of the predual and ideal structure of a $J B W^{*}$-triple. Math. Scand., 61:117-133, 1987.

[14] W. B. Johnson and J. Lindenstrauss. Handbook of the Geometry of Banach Spaces, Volumes 1 and 2. North Holland, 2001, 2003.

[15] J. Lindenstrauss and L. Tzafriri. Classical Banach Spaces I and II. Springer, Berlin-Heidelberg-New York, 1977, 1979.

[16] Matthias Neufang. On Mazur's Property and Property (X). 2002. preprint.

[17] H. Pfitzner. L-summands in their biduals have Pełczyński's property $\left(\mathrm{V}^{*}\right)$. Studia Math., 104:91-98, 1993.

[18] H. Pfitzner. A note on asymptotically isometric copies of $l^{1}$ and $c_{0}$. Proc. Amer. Math. Soc., 129:1367-1373, 2001.

[19] S. Sakai. $C^{*}$-Algebras and $W^{*}$-Algebras. Springer, Berlin-Heidelberg-New York, 1971.

[20] M. Talagrand. A new type of affine Borel functions. Math. Scand., 54:183$188,1984$.

Hermann Pfitzner

Université d'Orléans

BP 6759

F-45067 Orléans Cedex 2

France

e-mail: pfitzner@labomath.univ-orleans.fr 